\title{
IMPORTANCIA DE LA INVESTIGACIÓN CIENTÍFICA EN CIENCIAS FINANCIERAS Y CONTABLES
}

\author{
Dr. CARLOS A. EGÚSQUIZA PEREDA (*) \\ Lic. OLINDA EGÚSQUIZA PEREDA (**)
}

\section{INTRODUCCIÓN}

Frente a la necesidad permanente del contador público de aplicar la investigación científica en su formación profesional, actuación y desarrollo personal, será preciso diseñar y aplicar una adecuada Metodología de Investigación que permita dar respuesta a la preocupación frecuente de los profesionales y estudiantes de ciencias financieras y contables. Estas preocupaciones pueden ser resumidas en las siguientes interrogantes: ¿Qué, cómo, con qué, bajo qué, por qué y para qué investigar en ciencias financieras y contables? Es preciso compartir esta reflexión con otros profesionales, ya sean en su calidad de docentes universitarios en las facultades de ciencias contables de las distintas universidades.

Es cierto que uno de los cursos de formación profesional del contador público en algunas universidades del país es Metodología de la Investigación; sin embargo, el ejercicio de esta profesión se circunscribe a las áreas de Contabilidad, Auditoría, Gestión, Tributación, Finanzas, Computación, etc., no así en el área de Investigación Científica.
Algunos trabajos de investigación de prestigiosos profesionales de estas ciencias carecen de una metodología, que contribuya como aporte de experiencias y conocimientos de parte de sus autores.

En lo referente a los estudiantes universitarios, el curso de Metodología de Investigación está respondiendo sólo a la necesidad de graduación y titulación; mas no para aplicarlo en el ejercicio profesional futuro.

Los datos estadísticos demuestran que la participación de profesionales con este conocimiento en proyecto de investigación multidisciplinario en materia financiera y contable no son satisfactorios. Todas estas preocupaciones exigen la necesidad de diseñar una metodología de investigación $\mathrm{y}$, sobre todo, plantear estrategias de cambio de los paradigmas tradicionales de la currícula de estudios de la formación del profesional contable. Por otro lado, se debe enfatizar e incentivar hacia la investigación científica en todos los cursos desde el primer año de estudio.

$\left(^{*}\right)$ Director de la Unidad de Postgrado y Docente Principal de la Facultad de Ciencias Contables de la UNMSM.

${ }^{(* *)}$ Docente Auxiliar de la Facultad de Ciencias Contables de la UNMSM. 


\section{LA INVESTIGACIÓN EN CIENCIAS FINANCIERAS Y CONTABLES}

El punto de partida de la investigación científica es el problema de investigación, el cual puede ser factual y formal, teórico y empírico. No hay investigación científica si no hay un problema. El problema se define como el hecho o fenómeno cuyo comportamiento debe ser descrito, explicado y pronosticado. La contabilidad, como disciplina social, plantea un conjunto de interrogantes que deben ser respondidos por el hombre. Por lo tanto, cada aspecto teórico y práctico de la contabilidad es un tema o problema de investigación.

Una fuente de problemas es el marco teórico de la contabilidad, las finanzas, la auditoría, la tributación, el peritaje contable, la administración, etc. Existen otras fuentes importantes de problemas o temas de investigación contable, los mismos que se van conociendo con las experiencias, las vivencias de todo profesional contable durante el ejercicio de la profesión; aspectos de organización contable, aspectos de la tributación, de manejo financiero, de costos, de planeamiento, de administración, de educación, etcétera.

En este campo hay mucho que investigar y se debe practicar la actitud de observar la realidad; revisar minuciosamente el marco teórico contable y, sobre todo, responden con sęntido reflexivo, crítico y creativo a las necesidades de la sociedad globalizada.

Después de identificar, seleccionar y formular el problema, el siguiente paso será, ¿cómo investigar? La respuesta será el método de investigación a seguir. El método se refiere al conjunto de pasos, procedimientos, cambios que debe seguirse para lograr una meta.
El marco teórico sobre metodología de investigación es muy amplio y rico, se debe elegir entre aquellos que se adecúen a los objetivos del trabajo de investigación iniciada y a la naturaleza del problema contable a investigar. El método nos permite garantizar los resultados a los que arribaremos durante el proceso de investigación.

La formulación del problema es el punto de partida del trabajo científico. Dicho problema contable nos motiva a plantear un conjunto de proposiciones consistentes y apropiadas que le darán respuesta y serán la solución del mismo; éste es el paso de la formulación de la hipótesis.

Los sistemas hipotéticos en la investigación contable son muy variados, tal como lo son los problemas contables, pues a cada problema contable, financiero, económico, administrativo, tributario, etc., le corresponde un sinnúmero de respuestas y soluciones previsibles. Respuestas o hipótesis que deberán ser contrastadas con datos de la realidad y la teoría existente para comprobar su grado de verdad o de falsedad.

Para la comprobación será necesario construir sistemas hipotéticos no sólo en base al marco teórico; sino en base a variables. Dichas variables se definen como rasgos, características, peculiaridades, valores, propiedades, etc., del problema contablefinanciero y son innumerables. Por ejemplo, cada rubro financiero, cada concepto tributario, cada aspecto de la función contable, cada elemento de los sistemas contables, cada normatividad contable, la normatividad de la auditoría; constituyen variables cualitativas y cuantitativas, dependientes e independientes.

La naturaleza del problema científico contable es el parámetro para complementar el método científico con la elección, entre 
los existentes, de un método especial o específico. Este método especial nos permite realizar la contrastación teórica y empírica de las hipótesis.

Existen muchos métodos específicos, enumeraremos aquellos que pueden ser empleados en el campo de la investigación contable: El Método Descriptivo, Correlacional, Diagnóstico Evaluativo, Ex Post Ipso, Experimental, Estudios de Casos, etc.

El Método Descriptivo será aplicable en la etapa de descripción o caracterización de la empresa, indicando sus componentes y aspectos considerados problema contablefinanciero; además de señalar sus rasgos y características.

El Método de Diagnóstico Evaluativo se aplicará cuando se va a explicar las razones o causas de los problemas contables-financieros para medir los niveles de productividad, rentabilidad, eficiencia, eficacia, utilidad, solvencia, liquidez, etc.

El Método Correlacional se empleará en aquellos casos en los que es preciso demostrar el grado de correlación entre variables económico-financieras, contables y administrativas, mediante datos cuantitativos o de estadística inferencial. Por ejemplo, el grado correlacional entre la presión tributaria y la evasión tributaria, entre la política económica y el crecimiento empresarial, entre la rentabilidad y la producción, etc.

El Método de Estudios Evolutivos tiene como propósito conocer, desde la perspectiva de los datos estadísticos, la evolución histórica del problema contable, haciendo comparaciones de un periodo económico a otro. Por ejemplo, los referidos al estudio de los niveles de recaudación tributaria en un quinquenio, las tasas de crecimiento de la población económicamente activa du- rante el período 1995 a 2000 , el comportamiento de los presupuestos de una entidad pública, etc.

El Método Ex Post Ipso es el estudio descriptivo y explicativo de problemas, hechos o fenómenos contables, financieros, económicos, administrativos, educativos. Por ejemplo, se puede estudiar con este método el nivel de captación de tributos indirectos durante un año, el nivel de producción alcanzado durante los últimos cinco años, el rendimiento académico de los estudiantes en la Facultad de Ciencias Contables de la UNMSM en el año 2000, etc.

El Método Experimental, a través de la experimentación, nos permite conocer algunos problemas de comportamiento humano que se presentan en el quehacer contable y en la realización de actividades económico-financieras, administrativas, etc. Tales problemas pueden ser: la evasión tributaria y los sistemas de información y educación tributaria, la participación del contador público en la toma de decisiones, el rendimiento de los trabajadores en la empresa y las políticas de incentivos, etc.

El Método de Estudios de Casos es aquel que, a través de la reducción, de lo particular a lo general, permite resolver problemas de los sistemas empresariales, los mismos que se organizan en empresas privadas, públicas, microempresas, pequeña empresa, gran empresa, comercio, industria, servicio, exportación, etc.

El método de investigación más recomendable en las ciencias financieras contables, va a depender de la naturaleza del problema de investigación, de los objetivos del trabajo de investigación, del tipo de información requerida de la naturaleza de la hipótesis y del criterio del investigador, etc. Siendo el más recomendable el Método de Diagnóstico Evaluativo ya que abarca los tres 
niveles: descriptivo, explicativo y pronóstico, que garantizan en cierta forma la rigurosidad y el nivel científico que la comunidad científica y la sociedad en su conjunto esperan.

El método nos permite orientarnos en la tarea de obtener la información para contrastar la hipótesis. El investigador tendrá que contar, además, con un conjunto de instrumentos que le permitan obtener información, sistematizarla, tratarla y analizarla; es decir, valerse de las técnicas de investigación. Las técnicas de investigación más recomendables que se deben utilizar en la recopilación de la información requerida para la contrastación empírica y teórica de la hipótesis son: entrevistas, cuestionarios, observación, revisión de fuentes, test, focus y group, sociodramas, simulaciones, etc.

La supervivencia de las Ciencias Financieras y Contables en el siglo XXI dependerá en gran medida del trabajo científico desarrollado por sus profesionales. El trabajo científico debe constituir una actitud de vida diaria del Contador Público para lograr su desarrollo profesional, personal y contribuir a su desarrollo y de la sociedad totalmente globalizada.

\section{ASPECTO DE LA INVESTIGACIÓN EN CIENCIAS CONTABLES}

- El contenido de la investigación en las Ciencias Contables comprende dos aspectos:

-Investigación contable estructural.

-Investigación contable social.

\section{Investigación Contable Estructural}

Debe entenderse la búsqueda metódica, racional y objetiva de conocimientos que permitan la descripción, explicación y control de fenómenos de naturaleza financiera.

\section{Investigación Contable Social}

Entiéndase la búsqueda metódica, racional y objetiva de conocimientos que permitan la descripción, explicación, control, generalización y predicción de fenómenos biopsicosociales.

De cualquier manera, la investigación en ciencias contables, sea estructural o social, tiene presente el método de la investigación científica.

\section{EL INVESTIGADOR EN LAS CIENCLAS CONTABLES}

En su sentido lato, el investigador en ciencias contables es toda persona con carácter de estudiante, graduando o profesional de la contabilidad que efectúa investigaciones dentro de su disciplina. Estos trabajos de investigación pueden ser eventuales o más o menos habituales.

En sentido estricto, el investigador contable es todo contador público que efectúa trabajos de investigación de modo permanente dentro de su ámbito profesional.

El estudiante suele investigar para preparar una clase o presentar un trabajo, el graduando, para elaborar su seminario de investigación contable, o tesis profesional, y el contador público, para vencer obstáculos en su desempeño profesional, para mantener sus productos o servicios con aceptación en el mercado.

En cambio, el Contador Público-Investigador se dedica formalmente a la investigación ocupando plazas con este carácter en instituciones académicas y empresas. Cultiva, así, nuevas teorías de avanzada para tener éxito en un mundo globalizado. 
PERFIL DEL INVESTIGADOR EN

LAS CIENCIAS CONTABLES

1. Debe conocer y manejar el Método Cientifico.

2. Debe ser un profesional con ética, creativo, objetivo, realista y emprendedor.

3. Debe ser un profundo seguidor de la verdad, observador, reflexivo, abierto al cambio y capaz de desarrollar un agudo sentido crítico.

4. Debe ser capaz de captar problemas y poner a disposición de la sociedad los hallazgos de sus investigaciones con honestidad, consistencia y solidez.

5. Debe conocer profundamente los antecedentes, la situación que guardan las ciencias contables con el conocimiento universal.

6. Estará al día en materia de informes, textos y publicaciones propias de la profesión.

7. Estará capacitado para sistematizar y poseer el sentido del orden y la organización.

8. Debe ser mentor, sinergético, proactivo y sobre todo manejar comunicación asertiva.

9. Capacidad de análisis de cifras y símbolos en el proceso de la investigación.

10. Mostrará habilidad para expresarse con precisión y corrección de manera particular al emplear el lenguaje escrito.

\section{LIMITACIONES DE LA INVESTIGACIÓN EN LAS CIENCIAS CONTABLES}

Además, de las limitaciones de la investigación científica en general, como son la escasez de recursos, prejuicios socioculturales e intereses conservadores, la contabilidad carece de programas de investigación en sus cuadros académicos institucionales públicos o privados; o bien la investigación que existe es insuficiente o deficiente, fundamentalmente por las siguientes razones:

1. Indiferencias hacia las actividades de investigación de las ciencias contables. Suele pensarse que en la contabilidad todo está dicho. Que no hay nada por hacer. Se otorga mayor importancia a la docencia que a la investigación, sin reparar que la primera se nutre de la segunda y sólo así se puede contar con docentes proactivos, mentores y aprendientes; se tiene que efectuar investigación permanente para poder enfrentar el reto de la globalización y reingeniería del siglo XXI.

2. Alto sentido pragmático de los miembros de la profesión contable.

Las instituciones académicas suelen desechar la investigación por considerarla una inversión no recuperable. El profesional de la contabilidad aspira a actividades especialmente lucrativas. Existen pocas plazas de investigación contable, no siempre remuneradas; por el contrario, el costo de la investigación y el tiempo empleado en el proceso de la investigación, bajo el concepto moderno, debe considerarse una inversión.

Algunos autores atribuyen la carencia de reconocimiento científico, de la disciplina contable, a la falta de científicos contables ya que toda ciencia requiere de un número suficiente de inversionistas dedicados a las tareas particulares de la investigación en la difusión de sus descubrimientos. 


\section{ESTRUCTURA DE UN PROYECTO DE INVESTIGACIÓN CIENTÍFICO}

Título: « $\gg$

1.0 PLANTEAMIENTO DEL PROBLEMA:

1. Antecedentes y formulación del problema

2. Justificación e importancia

3. Definición del problema

4. Limitaciones

2.0 OBJETIVOS:

1. Generales

2. Específicos

3.0 MARCO TEÓRICO Y CONCEPTUAL:

1. Antecedentes de la investigación

2. Bases teóricas

3. Definición de términos básicos

4. Hipótesis

4.0 SISTEMA DE VARIABLES:

1. Determinación de variables

2. Propuestas de indicadores

5.0 METODOLOGÍA DE LA INVESTIGACIÓN:

1. Tipo y nivel de investigación

2. Cobertura del estudio:

a. Universo o población: muestra, unidades de análisis

b. Ámbito geográfico

c. Período de análisis

3. Fuentes, técnicas e instrumentos de recolección de datos

4. Procesamiento y presentación de datos

5. Análisis e interpretación de datos y resultados
6.0 ASPECTOS ADMINISTRATIVOS:

1. Cronograma de actividades

2. Asignación de recursos: humanos y materiales

3. Presupuesto o costo del proyecto

4. Financiamiento

- Bibliografía

- Anexos

- Esquema del informe de investigación

- Redacción del informe final de investigación 
ANGELES CABALLERO, César.

1998. La Tesis Universitaria. Investigación Elementos. $8{ }^{a}$ edición, Lima-Perú.

AVILA ACOSTA, Roberto.

1997. Introducción a la Metodología de la Investigación. lra. edición, Editorial Estudios y Ediciones S.A. Lima-Perú.

LAVADO ROJAS, Abad.

1998. Contabilidad y Finanzas. 1ra. edición, Editorial Las Hormigas E.1.R.L., Lima-Perú.

LAURA ELESKANO, Luz.

1996. Ciencias y Metodologias para iniciarse en la investigación. 1 ra. edición, San Marcos, Lima - Perú.

MENDEZ, Carlos.

1995. Guía para elaborar diseños de Investigación en Ciencias Económicas, Contables y Administrativas. Editorial Mc Graw-Hill. Inter-Americana,S.A. Colombia.

ELIZONDO LÓPEZ, Arturo.

1992. La Investigación Contable Significación y Metodología. Ediciones Contables y Administrativas S. A. México. 